\title{
Volume Modulated Arc Radiation Therapy: An Important Tool for
}

\section{Pediatric Radiation 0ncology}

\author{
Linda Ding ${ }^{1}$ Shannon Higgins ${ }^{1}$ | Maureen Britton RN ${ }^{1}$ | Joshua Taylor $^{1}$ | Julie Trifone ${ }^{1}$ | Marious \\ Croos $^{1}$ | Thomas Quinn ${ }^{1}$ | Bashera Nochomowitz ${ }^{1}$ | Linda O'Connor ${ }^{1}$ | Tracy Serrentino $^{1}$ | Stefanie \\ Lowas $^{2}$ | Naheed Usmani² | Neil Grossman² | Jason Shohet ${ }^{2}$ | Muriel Cleary ${ }^{3}$ | Jeremy Aidlen ${ }^{3}$ | \\ Michael Hirsch $^{2}$ | Salwa Khedr ${ }^{4}$ | Christine Wallace ${ }^{5}$ | Janaki Moni ${ }^{1}$ | Maryann Bishop-Jodoin ${ }^{1}$ | \\ Kenneth Ulin ${ }^{1}$ | Paul Rava ${ }^{1}$ | Shirin Sioshansi ${ }^{1}$ | Rashi Garg${ }^{1}$ | Jessica Yancey ${ }^{1}$ | TJ FitzGerald ${ }^{1}$
}

\section{*Correspondence: TJ FitzGerald}

Address: ${ }^{1}$ Department of Radiation Oncology, University of Massachusetts Medical School, Worcester, MA 01655, USA; 2Department of Pediatrics, University of Massachusetts Medical School, Worcester, MA 01655, USA; ${ }^{3}$ Department of Surgery, University of Massachusetts Medical School, Worcester, MA 01655, USA; ${ }^{4}$ Department of Pathology, University of Massachusetts Medical School, Worcester, MA 01655, USA; ${ }^{5}$ Department of Radiology, University of Massachusetts Medical School, Worcester, MA 01655, USA

e-mail $\bowtie:$ TJ.Fitzgerald@umassmemorial.org

Received: 04 November 2020; Accepted: 09 November 2020

Copyright: (C) 2020 Ding L. This is an open-access article distributed under the terms of the Creative Commons Attribution License, which permits unrestricted use, distribution, and reproduction in any medium, provided that the original work is properly cited.

\section{ABSTRACT}

Radiation therapy continues to be an important treatment application in pediatric oncology. There have been significant improvements in the application of radiation therapy in the past decade which can be directly applied to children and improve patient outcome relative to tumor control and ameliorate the late effects of management imposed on normal tissue. In this paper we present an application of radiation therapy using volume modulation arc therapy in a unique presentation of Ewing sarcoma. Multiple body segments were treated in a simultaneous manner with conformal avoidance of critical normal tissues including cardiac structures, hepatic parenchyma, and the remaining left kidney.

Keywords: Ewing's Sarcoma, Lung Metastasis, Radiation Therapy

\section{Introduction}

Radiation therapy continues to play an important role in pediatric oncology. Many primary disease areas including central nervous system, musculo-skeletal system, abdomen, and isolated sites of oligometastasis are treated with radiation therapy. Applying radiation therapy to clinical situations is a balance between successful tumor control and thoughtful effort to titrate the late effects of therapy.

There have been extraordinary changes in the application of radiation therapy over the past two decades. Historically with two-dimensional radiation therapy planning strategies, large segments of 
hepatic parenchyma would be included in the treatment field of a right-side Wilms tumor as well as inferior pulmonary volume in the right lung. For left-sided Wilms tumor radiation therapy, a large segment of cardiac anatomy would be included in the therapy field including left -sided pulmonary parenchyma. Whole lung radiation therapy for pulmonary metastasis requiring radiation therapy would include the entire cardiac anatomy in the therapy field and late effects on normal tissue became an unintended consequence of management. With the increasing effectiveness of chemotherapy, late effects from management are more visible and have left a significant impact on the health of survivors of childhood cancer.

Fortunately, changes in the delivery and application of radiation therapy can now be applied to pediatric oncology. Targeting is performed with volumetric tools and fluence profiles can be modulated with multileaf collimators to both augment dose to target and provide conformal avoidance to critical normal tissue structures. Modern image guidance tools including optical tracking are nimble and therapy can be image validated and executed in a few minutes including care of patients with complex geometries facilitating clinical management and patient care. These changes have had an important impact in the care of patients and have brought the field of radiation oncology forward with rapid speed.

We present a case report reflecting the modernization of radiation oncology. The patient is a 7year-old female who presented to our institution with an abdominal mass and image evidence of pulmonary lesions. Computer tomography suggested origin of the lesion to be in the right renal parenchyma consistent with Wilms tumor, stage 4 with pulmonary metastasis. At the time of abdominal surgery, tumor appeared to extend beyond the capsule of the renal parenchyma with enlarged lymph nodes removed. The final pathology, however, revealed unanticipated findings. The primary tumor was $12 \mathrm{~cm}$ in size and exhibited small round cell features with EWSR1 gene rearrangement consistent with Ewing sarcoma. Disease extended into the renal pelvis with extension into soft tissue at the renal sinus including involvement of the renal vein. Two lymph nodes were positive for metastasis, one in the right renal hilum and one in the left caval region. Next generation sequencing confirmed the EWSR1 gene rearrangement consistent with Ewing sarcoma. Pediatric and radiation oncology colleagues were preparing to treat the patient for a presumed Wilms tumor, however the biomarkers required an alteration in strategy requiring treatment be administered in a manner consistent with Ewing sarcoma protocols. From a radiation therapy perspective, this required a treatment strategy designed to treat both pulmonary and abdominal volumes in a comprehensive manner to higher radiation doses beyond what would be administered for Wilms tumor.

Chemotherapy was administered consistent with standard therapy protocols for Ewing sarcoma including Doxorubicin, Etoposide, Ifosfamide, Cyclophosphamide, and Vincristine with appropriate 
supportive medications including growth factor support, dexrazoxane, and mesna with radiation therapy included at the appropriate time point per protocol.

The challenge for the administration of radiation therapy was to deliver protocol appropriate dose to both the thorax and abdomen with each requiring segregate radiation doses and target volumes. Historically, these targets would be treated as separate volumes with individual plans. There would be a distinct disadvantage with this approach as individual plans treating the abdomen and thorax would require potential overlap of radiation therapy treatment fields increasing the risk of both short term and long-term injury. This technique is tacitly accepted in Wilms tumor as radiation doses delivered are often small by comparison to other disease groups. However, in a patient with Ewing sarcoma, control of disease with radiation therapy requires comprehensive treatment to high dose even in patients whose disease is resected, therefore a strategy to deliver dose to sites of disease requiring therapy would require a novel approach to both treat the disease and provide reasonable normal tissue conformal avoidance. At presentation, the size of the primary lesion created significant distortion of the liver (Fig. 1). Identifying areas at risk as the liver reestablished more traditional anatomical location was complex to achieve conformal avoidance of hepatic parenchyma and limit hepatic volume receiving high dose radiation therapy. The whole lung component of therapy would traditionally include the entire cardiac parenchyma with likely overlap to the abdominal volume. Modern therapy permitted an alternate approach to management.

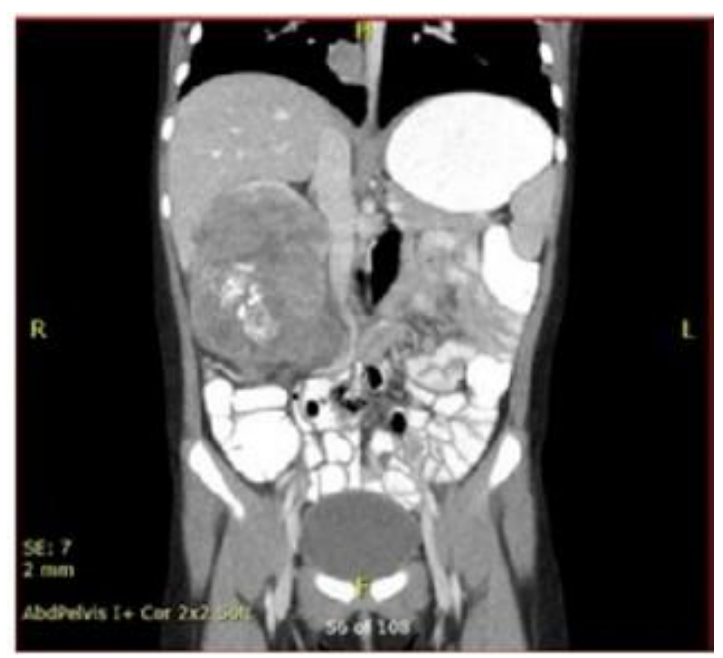

Figure 1: Abdominal imaging at presentation. Image courtesy of the Department of Radiology, University of Massachusetts Medical School

\section{Methods and Materials}

Unlike Wilms tumor where abdominal and pulmonary disease are often treated in sequence with thoracic radiation omitted when there is a complete response to chemotherapy, Ewing sarcoma 
radiation therapy requires comprehensive treatment to the operative site and regions of metastatic disease to the pulmonary parenchyma. Radiation therapy is imbedded into Ewing protocols at a later point than Wilms protocols when the situation does not need emergent radiation therapy. With volume modulated arc therapy (VMAT), we had the opportunity to evaluate integrating both targets into a single plan with simultaneous treatment delivery to all sites of disease. The plan also provided the opportunity to perform cardiac and hepatic conformal avoidance planning to limit both acute and late effects from management without intentional overlap of therapy fields. Surgical colleagues placed clips in the region where tumor abutted liver parenchyma. This proved to be extremely helpful as the liver returned to a more traditional anatomical location after surgery and identification of these areas of abutment otherwise would have been more challenging. This also provide an opportunity to optimize conformal avoidance to the bowel which occupied space vacated by disease.

The patient underwent four-dimensional simulation with disease targets drawn for full coverage of tumor sites including the original site of disease in the right kidney, para-renal tissues including soft tissue extension of disease at the renal pelvis, involved abdominal lymph node regions, and pulmonary parenchyma. All sites were treated in the single plan to 15 Gy (pulmonary dose) with a subsequent final phase treatment directed to the surgical resection site and draining abdominal lymph node regions to a dose of 45 Gy to meet bowel tolerance. Patient tolerated therapy without incident.

For dose volume comparison, the plan with VMAT was compared using dose volume histogram tools with a therapy plan that would have been applied with historical standards. Dose to critical structures including the heart and lung were compared. Statistical analysis was performed with a student $\mathrm{T}$ test.

\section{Results}

Fig. 2 demonstrates the comparison of radiation dose to both the liver and cardiac anatomy using both plans. As can be seen, radiation dose to both critical structures are significantly reduced using volume modulated arcs which provides an opportunity to exclude significant portion of the liver and heart from therapy. In comparison, the entire heart is receiving more than 15 Gy in the historical plan and less than $20 \%$ of the heart is receiving 20 Gy with the plan using volume modulated arcs. With the historical plan more than $50 \%$ of the liver is receiving 40 Gy and in the VMAT plan less than 10\% of the liver is receiving $40 \mathrm{~Gy}$. Marked improvement in liver dose/analysis is seen at nearly all radiation doses. All results are statistically significant with $\mathrm{p}<0.05$. 

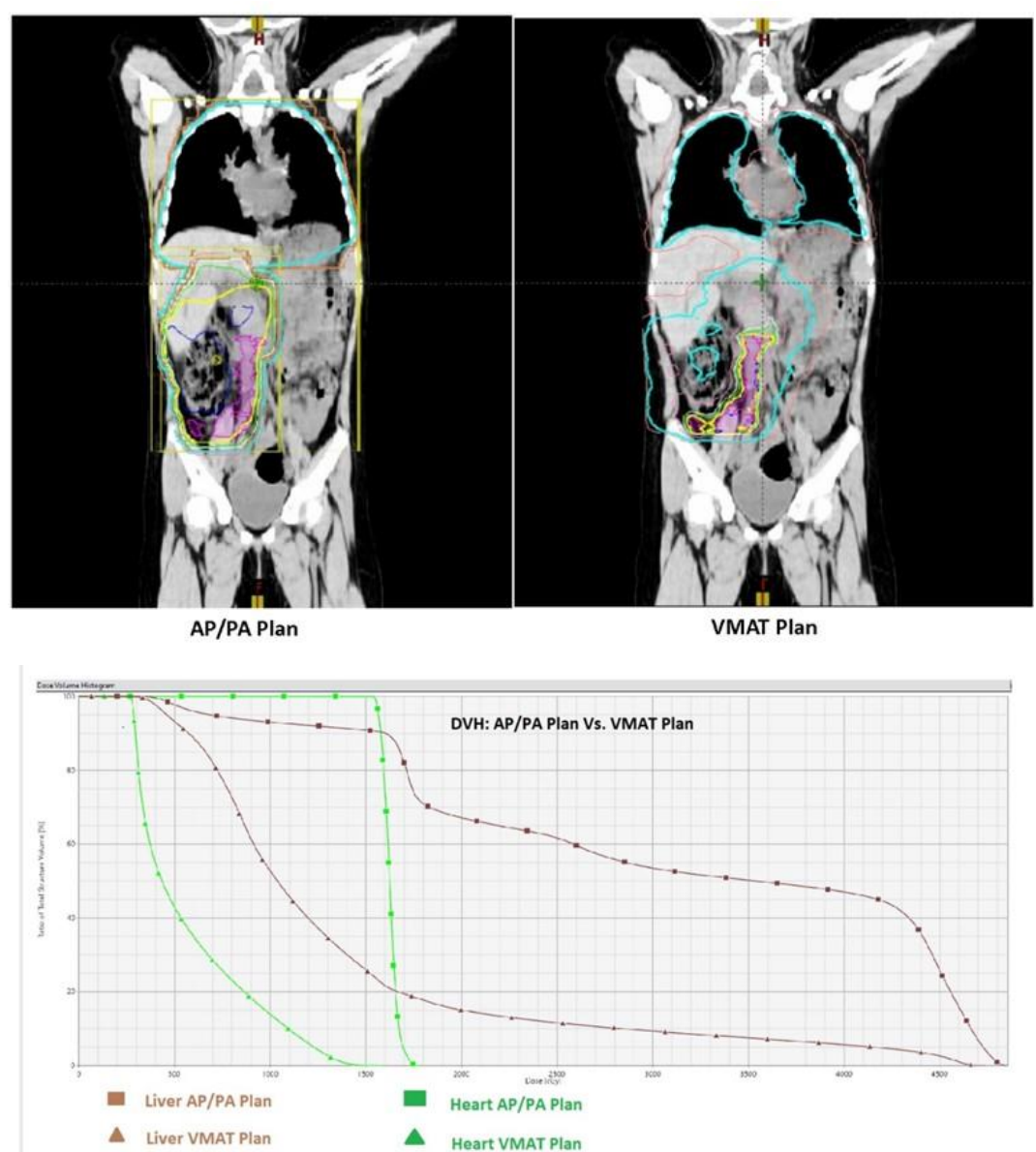

Figure 2: AP-PA plan, VMAT plan and the Dose Volume Histogram (DVH) for these plans. Image courtesy of the Department of Radiation Oncology, University of Massachusetts Medical School

The decrease in dose to the heart is seen in Fig. 3. Axial geometry of the radiation therapy treatment plan shows the marked improvement with conformal avoidance best seen in the central part of the heart including the region of both the aortic and mitral valves and left main coronary artery.

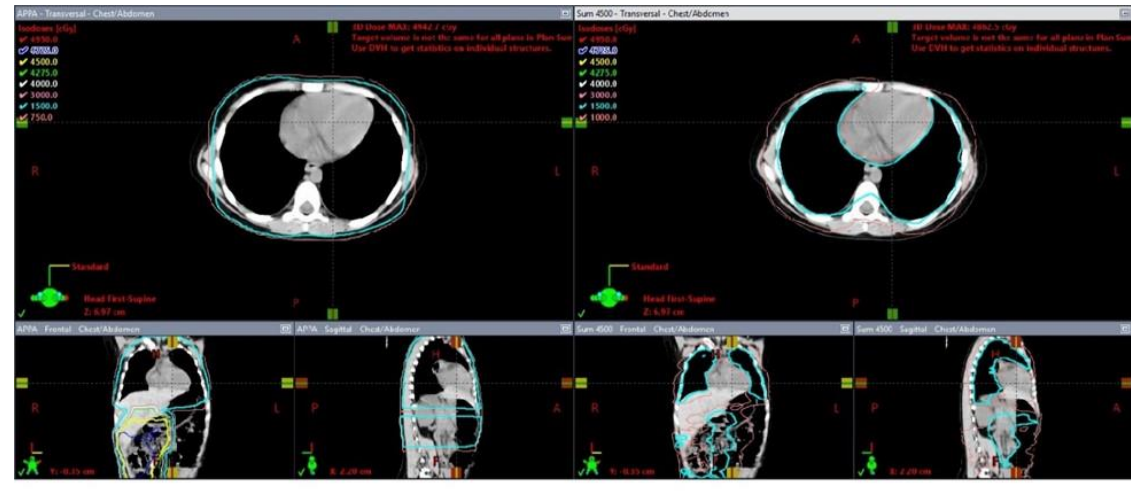

AP/PA Plan

VMAT Plan

Figure 3: Axial plan demonstration between AP-PA and VMAT. Images courtesy of the Department of Radiation Oncology, University of Massachusetts Medical School 


\section{Discussion}

There have been significant improvements in radiation therapy treatment delivery systems which have improved radiation dose to targets and limit radiation dose to normal tissue. VMAT has a unique advantage to more traditional therapy including static field intensity modulation as the gantry and multileaf collimators move in a synergistic manner and can "stop/start" radiation therapy treatment delivery and further shape radiation beam geometry around circumferential structures including the heart. Cardiac avoidance using four-dimensional therapy planning for whole lung radiation therapy was demonstrated by Kalapurikal and colleagues (Kalapurikal et al., 2019a; Kalapurikal et al., 2013; Kalapurikal et al., 2019b; Zhang et al., 2008; Kole et al., 2012; Scobioala and Eich, 2020; Suzuki et al., 2019) and has evolved as a good therapy strategy for treating patients who require whole lung therapy for metastatic disease. For this patient, we were able to extend this strategy and treat the patient to two regions with a comprehensive strategy with a single radiation therapy plan and apply a similar technique to limit dose to both the heart and the liver. Likewise, radiation dose was titrated across bowel and the remaining left kidney was untreated. Dose gradients were placed across vertebral bodies to promote further growth and development. The volume modulated therapy plan provided an opportunity for significant normal tissue sparing which would not have been possible without modern technology. The advantage of performing her care with a single plan is the elimination of the risk of overlap between fields delivered with separate plans and isocenters with optical tracking providing additional security managing motion during treatment execution.

One challenge to modern planning, however, is targeting. In this case, the primary tumor created an anatomical distortion and displacement of the liver and on a post-operative basis the liver had returned to a more traditional anatomical location. The challenge is to identify areas at risk in a structure which now resides in a different position, particularly in areas deemed at risk where tumor abutted the hepatic capsule which had rotated on a post-operative basis. Modern planning tools permit fusion of all diagnostic images into a planning study, however because of displacement of the liver, areas at risk are in a different location and not necessarily well defined by fusion objects. To limit radiation dose to liver, contours of therapy targets are more challenging and there is a risk of contouring too much tissue and too little tissue. This requires skill by those designing the plan and the participation of surgical and radiology colleagues in plan development is very important for generating an optimal outcome.

Modern radiation therapy can provide improved tumor targeting and conformal avoidance to normal tissue structures and will continue to support the management of children affected with various malignancies. As improvements in radiation therapy can be applied to patient care, outcome will 
improve in a parallel manner.

\section{Author Contributions}

Linda Ding PhD- Study concept/writing the paper/data collection; Maryann Bishop-Jodoin MEd- Data analysis/interpretation; TJ FitzGerald MD- Writing the paper/data collection/study design; Additional authors- Contributors

\section{References}

Kalapurikal JA, Gopalakrishnan M, Walterhouse DO, Rigsby CK, Rademaker A, Helenowski I, Kessel S, Morano K, Laurie F, Ulin K, Esiashvili N, Katzenstein H, Marcus K, Followill DS, Wolden SL, Mahajan A, Fitzgerald TJ. Cardiac-sparing whole lung IMRT in patients with pediatric tumors and lung metastasis: final report of a prospective multicenter trial. Int J Radiat Oncol Biol Phys 2019a; 103: 28-37.

Kalapurikal JA, Lee B, Bautista J, Rigsby C, Helenowski I, Gopalakrishnan M. Cardiac-sparing whole lung intensity modulated radiation therapy in children with Wilms tumor: Final report on technique and abdominal field matching to maximize normal tissue protection. Pract Radiat Oncol 2019b; 9: e62-e73.

Kalapurikal JA, Zhang Y, Kepka A, Zawislak B, Sathiaseelan V, Rigsby C, Gopalakrishnan M. Cardiac sparing whole lung IMRT in children with lung metastasis. Int J Radiat Oncol Biol Phys 2013; 85: 761-767.

Kole TP, Aghayere 0, Kwah J, Yorke ED, Goodman KA. Comparison of heart and coronary artery doses associated with intensity-modulated radiotherapy versus three-dimensional conformal radiotherapy for distal esophageal cancer. Int J Radiat Oncol Biol Phys 2012; 83: 1580-1586.

Scobioala S and Eich HT. Risk stratification of pulmonary toxicities in combination of whole ling irradiation and highdose chemotherapy for Ewing sarcoma patients with lung metastasis: a review. Strahlenther Onkol 2020; 196: 495-504.

Suzuki G, Ogata T, Aibe N, Yamazaki H, Yagyu S, Iehara T, Hosoi H, Yamada K. Effective heart-sparing whole lung irradiation using volumetric arc therapy: a case report. J Med Case Rep 2019; 13: 277.

Zhang X, Zhao K, Guerrero TM, McGuire SE, Yaremko B, Komaki R, Cox JD, Hui Z, Li Y, Newhauser WD, Mohan R, Liao Z. Four-dimensional computed tomography-based treatment planning for intensity modulated radiation therapy and proton therapy for distal esophageal cancer. Int J Radiat Oncol Biol Phys 2008; 72: 278-287. 